\section{Nefropatías secundarias a paraproteína: perfil clínico a partir de hallazgos histológicos}

\author{
JOAQUÍN JEREZ ${ }^{1}$, CRISTIÁN JUANET ${ }^{1}$, \\ JOAQUÍN SHARP ${ }^{1}$, DANIEL ERNST ${ }^{2}$, \\ GONZALO P. MÉNDEZ ${ }^{3}$, RODRIGO A. SEPÚLVEDA ${ }^{4}$
}

\section{Paraprotein-related kidney disease. Experience in 22 cases}

Background: One of the devastating consequences of monoclonal gammopathies is the development of end-stage kidney disease, which can be prevented with an early diagnosis. Renal involvement can be secondary to saturation of paraproteins with intratubular precipitation or the glomerular deposition of paraproteins with secondary inflammation and destruction. These conditions can also be associated with monoclonal gammopathies that do not meet hematological treatment criteria, called monoclonal gammopathies of renal significance (MGRS). Aim: To report a retrospective analysis of patients who underwent a renal biopsy and whose final diagnosis was a form of monoclonal gammopathy. Material and Methods: We reviewed the clinical and laboratory features and response to treatment of 22 patients aged $63 \pm 12$ years (55\% women) with a pathological diagnosis of a nephropathy associated with paraproteinemia. Results: The most common hematological diagnosis was amyloidosis in 50\% of patients, followed by cast nephropathy. The predominant clinical presentations were proteinuria (without nephrotic syndrome) and nephritic syndrome. Classic criteria such as erythrocyte sedimentation rate $>100 \mathrm{~mm} / \mathrm{h}$ and protein-albumin gap were unusual. Serum light chain quantification was the test with the best yield to detect paraproteins. Conclusions: In this group of patients, light chains tend to affect the kidney more commonly than heavy chains. The prognosis of multiple myeloma is much worse than MGRS.

(Rev Med Chile 2020; 148: 1059-1067)

Key words: Glomerulonephritis; Multiple Myeloma; Nephrotic Syndrome; Paraproteinemias; Proteinuria.

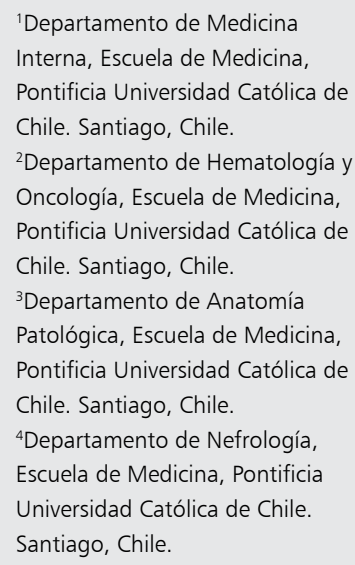

Trabajo no recibió financiamiento. Los autores declaran no tener conflictos de interés.

Recibido el 24 abril de 2020, aceptado el 3 de septiembre de 2020.

Correspondencia a:

Dr. Rodrigo Sepúlveda Palamara

Departamento de Nefrología.

Diagonal Paraguay 362, piso 4.

Santiago, Chile.

Código postal: 8330077

rrsepulveda@uc.cl

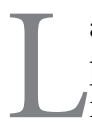

as gammapatías monoclonales corresponden a una entidad hematológica cuya importancia ha ido en ascenso. Se estima que 3\%-4\% de la población mayor de 50 años tendrían una gammapatía monoclonal de significado incierto (MGUS), con una prevalencia que aumenta con la edad ${ }^{1}$. Tanto el mieloma múltiple (MM) como la amiloidosis por cadenas livianas (amiloidosis AL) fueron los primeros trastornos de células plasmáticas en donde se pudo determinar que el depósito de inmunoglobulina generaría un daño tisular ${ }^{2,3}$.

El término de gammapatía monoclonal de significado renal (GMSR) fue acuñado en el año $2012^{4}$, y se describió en pacientes con compromiso por enfermedad o desorden monoclonal diagnosticado a nivel renal mediante biopsia, sin cumplir criterios de tratamiento para una neoplasia plasmocitaria o de células B de base (ejemplo: MGUS, MM asintomático, macroglobulinemia de 
Waldeström indolente). La forma más conocida de nefropatía asociada a una paraproteína es el "riñón de mieloma" (RM), en donde el exceso de una paraproteína, generalmente cadena liviana, filtra por un glomérulo sano y luego precipita por sobresaturación a nivel intratubular. El RM produce falla renal y se presenta en pacientes que cumplen criterios para mieloma múltiple sintomático. En este caso, el nivel de paraproteína es tan alto que puede ser detectado por las técnicas menos sensibles de diagnóstico. Sin embargo, en la GMSR el compromiso renal tiende a ser distinto, ya que son las propiedades físico-químicas de la paraproteína lo que determina su depósito en alguna estructura del nefrón (glomérulo o células tubulares), ocasionando múltiples formas de daño con diversas presentaciones clínicas y morfológicas. Por lo anterior, no se requiere una cantidad excesiva de paraproteína para generar este trastorno, se acompaña frecuentemente de daño glomerular y se necesitan métodos diagnósticos más sensibles para detectar la escasa cantidad de paraproteína ${ }^{5}$. Las principales formas histológicas de afección renal en GMSR son: Amiloidosis AL (de cadena liviana $[\mathrm{CL}]$ ) o menos frecuente ami- loidosis AH (de cadena pesada [CP]), depósito glomerular de inmunoglobulinas monoclonales con o sin glomerulonefritis proliferativa (con mayor frecuencia IgG monoclonal), depósitos de CL y/o CP en membranas basales glomerulares y tubulares, otras formas de depósitos organizados (glomerulonefritis inmunotactoide, crioglobulinemias), cristalización tubular proximal de cadenas livianas, glomerulopatía por inducción de alteración en vía alterna de complemento (C3), entre las principales (Tabla 1$)^{6,7}$.

Como las GMSR no tienen niveles detectables de paraproteína o un clon neoplásico identificado que correspondan a una indicación de tratamiento, es el compromiso renal lo que comanda la indicación terapéutica ${ }^{8}$. Se ha estimado que $6 \%$ de los pacientes en quienes inicialmente se sospecha MGUS tendría daño renal ${ }^{9}$. Es muy importante mantener un alto índice de sospecha, ya que los pacientes con GMSR tienen peor sobrevida que pacientes con $\mathrm{MGUS}^{9}$ y mayor riesgo de evolución a $\mathrm{MM}^{10}$. El tratamiento de la GMSR mejora la sobrevida renal ${ }^{11,12}$, y si este es precoz, evitaría el daño renal irreversible ${ }^{13,14}$. La misma experiencia se ha replicado en MM. En pacientes en seguimiento por

\section{Tabla 1. Nefropatías asociadas a paraproteínas ${ }^{6,7}$}

\section{Depósitos glomerulares organizados:}

Amiloidosis por CL y/o CP (depósito organizado fibrilar en placas de ß-amiloide)*

Crioglobulinemia tipo I (depósito organizado microtubular) ${ }^{\dagger}$

Glomerulopatía inmunotactoide (depósito organizado microtubular) ${ }^{\dagger}$

Glomerulopatía fibrilar (depósito organizado fibrilar) ${ }^{\dagger}$

\section{Depósitos glomerulares no organizados:}

Enfermedad por depósito de inmunoglobulina monoclonal (MIDD): puede ser de cadena liviana (LCDD), por depósito de cadena pesada (HCDD) o ambas (LHCDD)*

Enfermedad por depósito de inmunoglobulina monoclonal con glomerulonefritis proliferativa asociada (PGNMID) ${ }^{\dagger}$

Paraproteína inductora de microangiopatía trombótica glomerular:

Lesión endotelial directa (inducida por paraproteína) o indirecta (inducida por secreción de citoquinas) ${ }^{\dagger}$

Paraproteína con actividad anti-Factor $\mathrm{H}$ del complemento ${ }^{\dagger}$

\section{Paraproteína inductora de glomerulopatía:}

Glomerulopatía membranosa por IgG3 Kappa monoclonal contra receptor de fosfolipasa A2

Glomerulonefritis $\mathrm{C3}^{\dagger}$

Enfermedad por depósitos densos ${ }^{\dagger}$

\section{Lesión túbulo-intersticial:}

Precipitación intratubular de cadenas livianas o riñón de mieloma (característico del MM)

Síndrome de Fanconi por cristalización de paraproteína en célula tubular proximal

Atrofia tubular por acumulación amorfa de paraproteína en célula tubular proximal

Atrofia tubular por cristalización de paraproteínas en histiocitos intersticiales

*Microscopía óptica: Esclerosis nodular mesangial. `Microscopía óptica: Glomerulonefritis mesangiocapilar. CL: cadena liviana; CP: cadena pesada; MM: mieloma múltiple. 
MGUS con evolución a MM, el tratamiento precoz logra mejores resultados ${ }^{15}$. Debido a lo anterior, es perentorio generar evidencia respecto a las formas de presentación de estas patologías a nivel renal para un estudio adecuado y un diagnóstico y tratamiento oportunos.

\section{Metodología}

Se realizó un estudio de cohorte retrospectivo en pacientes adultos de nuestra institución que requirieron estudio histopatológico renal entre los años 2013 y 2017, con diagnóstico final de alguna forma de nefropatía asociada a paraproteínas. El objetivo principal fue describir y caracterizar el perfil clínicopatológico de estos pacientes. Específicamente, se evaluaron variables clínicas, de laboratorio, hallazgos morfológicos y pronóstico. No se analizaron las alteraciones citogenéticas por cariotipo o hibridación fluorescente in situ (FISH), debido a que no están disponibles en nuestra institución. Como objetivos secundarios, se analizaron los resultados del tratamiento, incluyendo la tasa de respuesta global y la mediana de sobrevida global.

El examen histológico renal incluyó microscopía óptica, de inmunofluorescencia y electrónica. La evaluación de gammapatía monoclonal fue realizada mediante estudios de electroforesis e inmunofijación de proteínas en sangre u orina, medición de CL séricas y estudio medular asociado a citometría de flujo de 8 colores y sensibilidad de $10^{-4}$ (BD FACSCanto ${ }^{\mathrm{TM}}$ II, BD Biosciences, Becton, Dickinson and Co., NJ, USA). El diagnóstico, la etapificación y la evaluación de respuesta a tratamiento se realizó de acuerdo con los criterios del International Myeloma Working Group de $2014^{16,17}$. La definición y clasificación de GMSR se realizó acorde a lo reportado en la literatura ${ }^{4-6}$. Se definieron como formas de presentación clínica renal: falla renal aislada (por una creatinina persistentemente elevada), proteinuria no nefrótica (PNN: $300 \mathrm{mg} / \mathrm{d}$ a $3,5 \mathrm{~g} / \mathrm{d}$ o equivalente), proteinuria en rango nefrótico (PN: $>3,5 \mathrm{~g} / \mathrm{d}$ ), síndrome nefrótico (SNo: proteinuria en rango nefrótico asociado a edema e hipoalbuminemia), y nefritis o síndrome nefrítico (SNi: hematuria glomerular asociado o no a proteinuria, hipertensión arterial y/o deterioro de la función renal $)^{18}$. La información clínica fue obtenida desde registros médicos electrónicos y registro nacional de defunciones.
El análisis de sobrevida se realizó mediante Kaplan-Meier y Log-rank test. Análisis bivariados se realizaron con pruebas no paramétricas: Mann-Whitney, Fisher y correlación de Spearman. Se utilizó software Stata v.14 y GraphPad Prism. Se consideró significativo un valor $\mathrm{p}<0,05$. Este estudio fue aprobado por el Comité Ético Clínico de la Facultad de Medicina de la Pontificia Universidad Católica de Chile.

\section{Resultados}

Fueron analizados un total de 22 pacientes; $54,6 \%$ de sexo femenino. La mediana de seguimiento fue de 2,5 años. La edad promedio al momento de la biopsia renal fue 63 años $( \pm 12,1)$. En

Tabla 2. Características pacientes

\begin{tabular}{|c|c|}
\hline Datos demográficos & $\begin{array}{l}\text { Promedio o fre- } \\
\text { cuencia (DE o n) }\end{array}$ \\
\hline $\begin{array}{l}\text { Edad } \\
\text { Mujeres }\end{array}$ & $\begin{array}{l}63 \text { años }( \pm 12,1) \\
54,6 \% \quad(n=12)\end{array}$ \\
\hline $\begin{array}{l}\text { Comorbilidades } \\
\text { HTA } \\
\text { DM2 } \\
\text { ERC } \\
\text { Cáncer }\end{array}$ & $\begin{array}{r}45,45 \%(n=10) \\
9,09 \%(n=2) \\
4,54 \%(n=1) \\
22,72 \%(n=5)\end{array}$ \\
\hline $\begin{array}{l}\text { Falla renal } \\
\text { Sin falla renal } \\
\text { Con falla renal } \\
\text { Requerimiento diálisis }\end{array}$ & $\begin{array}{ll}28,6 \% & (n=6) \\
71,4 \% & (n=15) \\
28,6 \% & (n=6)\end{array}$ \\
\hline $\begin{array}{l}\text { Cuadro clínico nefrológico } \\
\text { Proteinuria no nefrótica aislada } \\
\text { Proteinuria nefrótica aislada } \\
\text { Síndrome nefrótico } \\
\text { Nefritis } \\
\text { Falla renal aislada }\end{array}$ & $\begin{aligned} 28,6 \% & (n=6) \\
23,8 \% & (n=5) \\
9,5 \% & (n=2) \\
33,3 \% & (n=7) \\
4,8 \% & (n=1)\end{aligned}$ \\
\hline $\begin{array}{l}\text { Laboratorio } \\
\text { Creatinina }(\mathrm{mg} / \mathrm{dL}) \\
\text { Hemoglobina }(\mathrm{g} / \mathrm{dL}) \\
\text { Glóbulos blancos }\left(\mathrm{n} / \mathrm{mm}^{3}\right) \\
\text { Plaquetas }\left(10^{3} \mathrm{n} / \mathrm{mm}^{3}\right) \\
\text { VSG }(\mathrm{mm} / \mathrm{h}) \\
\text { LDH }(\mathrm{U} / \mathrm{L}) \\
\text { Proteínas totales }(\mathrm{g} / \mathrm{dL}) \\
\text { Albúmina }(\mathrm{g} / \mathrm{dL}) \\
\text { Gradiente albúmino-proteico }(\mathrm{g} / \mathrm{dL}) \\
\text { Proteinuria }(\mathrm{g} / 24 \mathrm{~h}) \\
\text { Hematuria }\end{array}$ & $\begin{aligned} & 3,5( \pm 3,9) \\
& 10,8( \pm 3,1) \\
& 7.562( \pm 2.290) \\
& 240( \pm 84) \\
& 61,3( \pm 43,7) \\
& 206 \quad( \pm 59) \\
& 6,04( \pm 1) \\
& 3,3 \quad( \pm 0,8) \\
& 2,7 \quad( \pm 0,7) \\
& 3,6 \quad( \pm 2,4) \\
& 35 \%(\mathrm{n}=7)\end{aligned}$ \\
\hline
\end{tabular}

DM2: diabetes mellitus tipo 2; ERC: enfermedad renal crónica; HTA: hipertensión arterial; LDH: lactato deshidrogenasa; VSG: velocidad de sedimentación globular. 
la Tabla 2 se resumen las características clínicas y de laboratorio en la población estudiada. Destaca una alta prevalencia de cáncer previo $(27 \%)$. El $71,4 \%$ de los pacientes tenía falla renal al momento del diagnóstico y la mayoría poseía hallazgos compatibles con nefritis y grados variables de proteinuria. El 28,6\% de los pacientes requería terapia de reemplazo renal al diagnóstico. Respecto a los hallazgos de laboratorio, destaca que solo 18,2\% presentaba velocidad de sedimentación globular (VSG) > $100 \mathrm{~mm} / \mathrm{h}$ y solo $22,7 \%$ presentó un gradiente albúmino-proteico $(\mathrm{GAP})>3,5 \mathrm{~g} / \mathrm{dL}$.

De los 22 pacientes, en $50 \%(n=11)$ el diagnóstico hematológico final fue mieloma múltiple; de estos, 72,7\% tenía falla renal (Tabla 3). El 31,8\% $(\mathrm{n}=7)$ tenía una GMSR y en ellos la prevalencia de falla renal fue $85,7 \%$. Un paciente (sin falla renal al momento del diagnóstico) presentó linfoma no Hodgkin (LNH) y en 3 pacientes $(13,6 \%)$ no fue posible establecer el diagnóstico medular. No hubo diferencia estadística en la prevalencia de falla renal según diagnóstico medular $(\mathrm{p}=0,36)$.

En cuanto al diagnóstico renal histopatológico: $45,6 \%$ de las biopsias presentaron amiloidosis renal; $22,7 \%$ riñón de mieloma; $13,6 \%$ enfermedad por depósito de inmunoglobulina monoclonal con glomerulonefritis proliferativa asociada (PGNMID); 13,6\% enfermedad por depósito de inmunoglobulina monoclonal (MIDD) y 4,5\% tubulopatía por cristalización de paraproteína. De los pacientes con amiloidosis renal $(\mathrm{n}=10)$, $40 \%$ tenía un MM, 40\% una GMSR y en $1(10 \%)$ LNH. Los pacientes con amiloidosis renal tenían preferentemente CL Lambda en la inmunofluorescencia. El tamaño promedio de fibras amiloides fue $8,2 \mathrm{~nm}( \pm 1,2)$. En todos los pacientes con MIDD o PGNMID, la CL presente en los depósitos era Kappa $(p=0,016)$. Todos los casos con MIDD correspondían a enfermedad por depósito de CL (LCDD) asociada a Kappa. Los 3 pacientes con PGNMID presentaron glomerulonefritis por depósito de IgG Kappa. Un paciente presentó cristalización proximal de paraproteína Kappa con síndrome de Fanconi y falla renal.

Los pacientes con RM, además de tener deterioro de la función renal, se presentaron tanto con PNN y PN (no hubo presentaciones con falla renal aislada ni SNo). Los pacientes con amiloidosis se presentaron principalmente con $\mathrm{PN}, \mathrm{PNN}$ y SNo. Aquellos con MIDD, clínicamente tenían SNi o falla renal aislada. Todos los pacientes con PGNMID se presentaron con SNi. Finalmente, la totalidad de

Tabla 3. Resumen de estudio histológico renal y medular

\begin{tabular}{|c|c|c|c|c|}
\hline Diagnóstico renal & Frecuencia & Depósito renal & $\begin{array}{l}\text { Paraproteína en } \\
\text { sangre/orina }\end{array}$ & $\begin{array}{c}\text { Diagnóstico } \\
\text { medular }\end{array}$ \\
\hline Amiloidosis AL & $40,9 \%(n=9)$ & $\begin{array}{ll}\text { Lambda } & (\mathrm{n}=8) \\
\text { Kappa } & (\mathrm{n}=1)\end{array}$ & $\begin{array}{ll}\text { Lambda aislado } & (\mathrm{n}=7) \\
\text { IgA Lambda } & (\mathrm{n}=1) \\
\text { IgG Lambda } & (\mathrm{n}=1)\end{array}$ & $\begin{array}{ll}\text { MM } & (\mathrm{n}=4) \\
\text { GMSR } & (\mathrm{n}=3) \\
\text { LNH } & (\mathrm{n}=1) \\
\mathrm{s} / \mathrm{d} & (\mathrm{n}=1)\end{array}$ \\
\hline Amiloidosis $\mathrm{AL} \mathrm{AH}$ & $4,5 \%(n=1)$ & IgG Kappa & IgG Kappa & GMSR \\
\hline MIDD (100\% LCDD) & $13,6 \%(n=3)$ & $(n=3)$ & $\begin{array}{ll}\text { Kappa aislado } & (\mathrm{n}=1) \\
\text { IgA Kappa } & (\mathrm{n}=1) \\
\text { IgG Kappa } & (\mathrm{n}=1)\end{array}$ & $\begin{array}{ll}\operatorname{MM} & (n=1) \\
\operatorname{GMSR} & (\mathrm{n}=2)\end{array}$ \\
\hline PGNMID & $13,6 \%(n=3)$ & IgG Kappa $(\mathrm{n}=3)$ & IgG Kappa & $\begin{array}{l}\text { GMSR }(n=1) \\
s / d \quad(n=2)\end{array}$ \\
\hline Tubulopatía por cristales & $4,5 \%(n=1)$ & Kappa & Kappa aislado & $\mathrm{MM}$ \\
\hline Riñón de mieloma & $22,7 \%(n=5)$ & $\begin{array}{ll}\text { Kappa } & (\mathrm{n}=3) \\
\text { Lambda } & (\mathrm{n}=2)\end{array}$ & $\begin{array}{l}\text { Kappa aislado } \quad(n=3) \\
\text { Lambda aislado }(n=2)\end{array}$ & $\mathrm{MM} \quad(n=5)$ \\
\hline
\end{tabular}

AH: amiloidosis de cadena pesada; AL: amiloidosis de cadena liviana; GMSR: gammapatía monoclonal de significado renal; LCDD: enfermedad por depósito de cadena liviana; LNH: linfoma no Hodgkin; MIDD: enfermedad por depósito de inmunoglobulina monoclonal; MM: mieloma múltiple; PGNMID: enfermedad por depósito de inmunoglobulina monoclonal con glomerulonefritis proliferativa; s/d: sin diagnóstico. 
Tabla 4. Rendimiento de estudios de paraproteínas

\begin{tabular}{|lcc|}
\hline Exámenes & Realizado & Sensibilidad \\
\hline $\begin{array}{l}\text { Electroforesis de proteínas } \\
\text { en sangre }\end{array}$ & $59,10 \%$ & $46,15 \%$ \\
\hline $\begin{array}{l}\text { Inmunofijación sangre } \\
\text { Inmunofijación orina }\end{array}$ & $68,10 \%$ & $80 \%$ \\
\hline $\begin{array}{l}\text { Cadenas livianas libres en } \\
\text { sangre }\end{array}$ & $42,70 \%$ & $56,30 \%$ \\
\hline
\end{tabular}

los pacientes con presentación clínica de síndrome nefrótico $(9,1 \%)$, tuvieron una amiloidosis renal.

En la población estudiada, la inmunofijación en sangre tuvo mejor rendimiento diagnóstico que en orina, aunque no fue significativo $(\mathrm{p}=0,07)$ (Tabla 4). En tres casos se detectó la paraproteína por inmunofijación en sangre y no en orina, al contrario, en solo un caso fue detectada solo en orina y no en sangre. La medición de CL en sangre resultó alterada en 100\% de los pacientes. Respecto al diagnóstico histológico en esta serie, destaca que, independiente de la morfología del daño renal, siempre hubo una CL monoclonal implicada, mientras que las CP solo se presentaron en los casos de PGNMID (IgG Kappa a nivel glomerular) y un caso de amiloidosis AL más AH (Tabla 3). El diagnóstico más frecuente en nuestra serie fue amiloidosis $\mathrm{AL}$; donde el componente monoclonal siempre correspondió a CL Lambda. A su vez, destaca que LCDD siempre estuvieron asociadas a cadenas Kappa. En el RM había indistintamente CL Kappa o Lambda. Al comparar el componente monoclonal de depósito tisular y el componente monoclonal detectado en sangre u orina, se aprecia que $42,3 \%$ de los pacientes presentaba un componente monoclonal de $\mathrm{CP}$, pero solo $18,2 \%$ tenía una $\mathrm{CP}$ a nivel renal.

En los pacientes con diagnóstico hematológico-medular establecido $(82,6 \%)$ fue posible describir su tratamiento y respuesta, en los restantes, por motivos previsionales, su diagnóstico hematológico y tratamiento se realizó en otros centros. El tiempo entre el diagnóstico e inicio de tratamiento tuvo una mediana de 1,8 meses. La mayoría $(61,1 \%)$ recibió inducción con ciclofosfamida-bortezomib-dexametasona (CyBorD), seguido de melfalán-prednisona-talidomida (MPT; $27,7 \%$ ) y el paciente con $\mathrm{LNH}$ recibió $\mathrm{R}-\mathrm{CHOP}$.
Excluyendo al paciente con $\mathrm{LNH}$ que no respondió a tratamiento y falleció a los 9 meses, la tasa de respuesta global fue de $76,5 \%$ (respuesta completa [RC] 61,5\%; respuesta parcial muy buena [RPMB] 15,4\%; respuesta parcial, 23,01\%). No hubo diferencias significativas en tasa de respuesta global entre ambos esquemas ( 80 versus $81,2 \%$ $\mathrm{p}=0,70) \mathrm{y}$, pese a tener mayor grado de respuesta (RC y RPMB) con esquema CyborD, 72,7\% versus $40 \%$ MPT, no hubo significancia estadística $(\mathrm{p}=0,24)$. Tras la inducción, en cinco pacientes $(23,8 \%)$ se realizó trasplante autólogo de precursores hematopoyéticos. Cuatro pacientes lograron $\mathrm{RC}$ estricta, con una mediana de seguimiento de 2,02 años, de los cuales tres tenían diagnóstico de GMSR y un paciente MM.

De los 19 pacientes que recibieron tratamiento, inicialmente seis $(31,6 \% ; 4$ con MM y 2 con GMSR) se presentaron con necesidad de hemodiálisis. De estos pacientes, luego del tratamiento $\mathrm{y}$ al final del seguimiento, solo 2 persistieron con dependencia de hemodiálisis (ambos con MM).

El análisis de sobrevida global evidencia que la mediana de sobrevida no fue alcanzada en la muestra total (Figura 1). Sin embargo, al subdividir la muestra por diagnóstico renal histológico se aprecian tres tendencias: amiloidosis, que presentaron una alta mortalidad precoz para luego detenerse; RM que presentaron una mediana de sobrevida en torno a los 40 meses, y pacientes con MIDD y PGNMID con $100 \%$ de sobrevida durante el período de seguimiento (Log-rank p =0,58). Respecto a la sobrevida según el diagnóstico hematológico; los pacientes con GMSR tuvieron mejor sobrevida que aquellos con MM (Log-rank p = 0,02). Entre los pacientes con amiloidosis por GMSR versus MM, no hubo diferencias de sobrevida (Log-rank $p=0,93$ ). Finalmente, no se reportaron diferencias en sobrevida con el uso de trasplante autólogo $(\log$-rank $\mathrm{p}=0,48)$.

\section{Discusión}

Los reportes latinoamericanos de nefropatías asociados a gammapatías monoclonales son escasos $^{19,20}$. En nuestra población, la forma de manifestación renal de las GMSR fue variada, destacando el compromiso inflamatorio glomerular, la presencia de proteinuria y, frecuentemente, deterioro de la velocidad de filtración glomerular. 


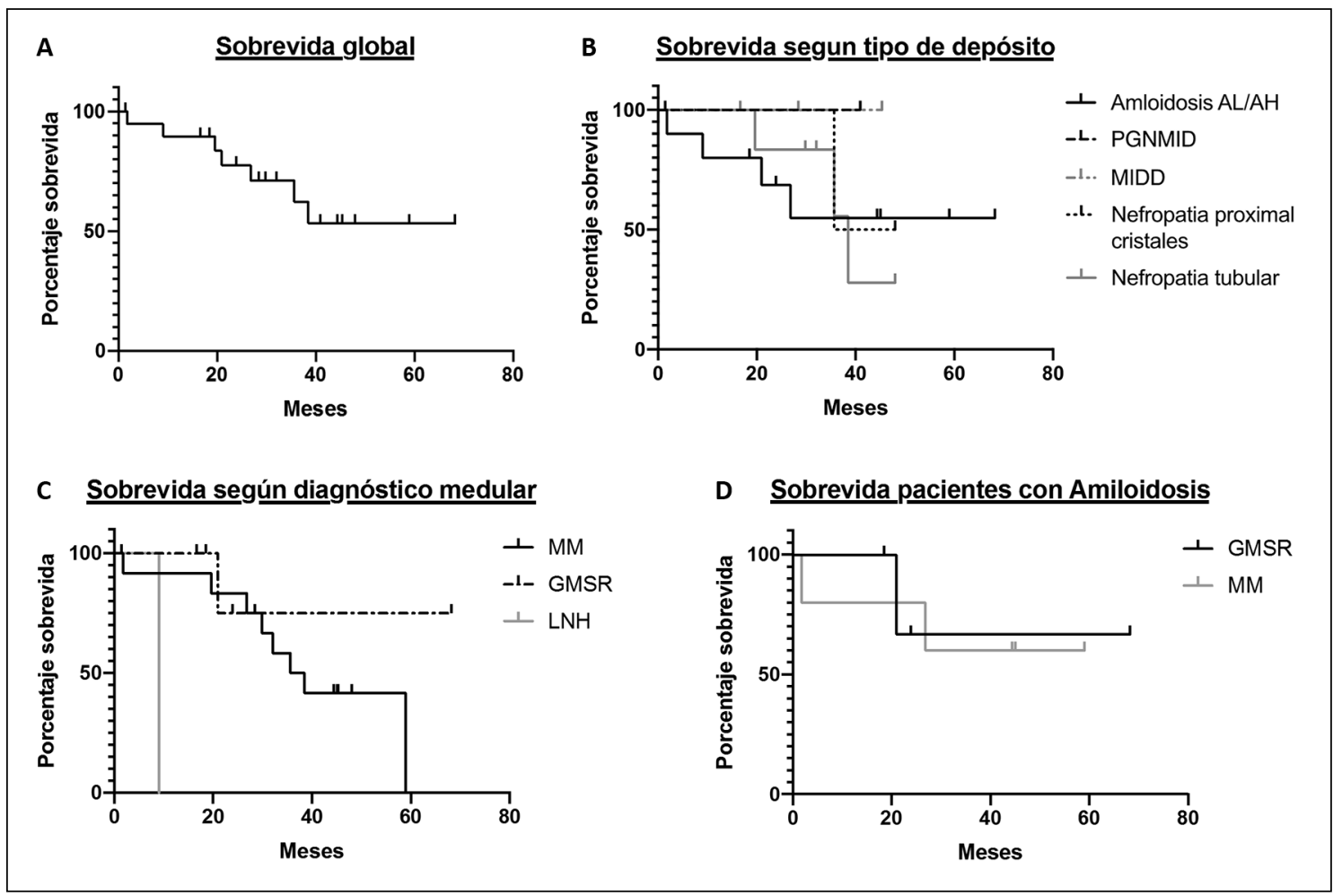

Figura 1. Análisis de sobrevida. Análisis de sobrevida global en muestra completa (A), según subtipo de histopatología renal (B) (Log-rank $p=0,58)$, según subtipo de diagnóstico hematológico (C) (Log-rank $p=0,02)$ y en pacientes con amiloidosis según su diagnóstico medular de base (D) (Log-rank $\mathrm{p}=0,93)$. AH: amiloidosis de cadena pesada; AL: amiloidosis de cadena liviana; GMSR: gammapatía monoclonal de significado renal; LNH: linfoma no Hodgkin; MIDD: enfermedad por depósito de inmunoglobulina monoclonal; MM: mieloma múltiple; PGNMID: glomerulonefritis proliferativa con depósitos de inmunoglobulina monoclonal.

La presentación como falla renal aislada es poco frecuente en concordancia con lo reportado en otras series $^{21,22}$. La edad promedio de diagnóstico también es la habitualmente descrita. Llama la atención la alta prevalencia de cáncer como antecedente e, interesantemente, no se describe en la literatura que los trastornos plasmocitarios tuvieran relación con uso de quimioterapia previa, a diferencia de leucemia mieloide aguda o síndromes mielodisplásicos.

En cuanto a las alteraciones presentes en el estudio de laboratorio general, destacamos que 40,9\% de los pacientes se presentaron con una VSG $<50$ $\mathrm{mm} / \mathrm{h}$ y que ninguno tenía un GAP $>4 \mathrm{~g} / \mathrm{dL}$. Esto se explica por la escasa cantidad de paraproteína producida por el clon celular anormal y por la alta frecuencia de CL exclusivas, que presentan un menor peso molecular. Así, se debe seguir sospe- chando una gammapatía monoclonal, aunque no exista elevación importante de VSG o GAP.

El compromiso histopatológico renal más frecuente fue amiloidosis renal, y luego, el RM. Esto se debe a una subrepresentación del RM, ya que cuando el diagnóstico de MM y proteinuria por cadenas livianas son evidentes, es posible prescindir de la biopsia renal ${ }^{23}$. Sin embargo, la nefropatía asociada a paraproteínas sensiblemente más frecuente es el RM ${ }^{5}$. De los pacientes con amiloidosis, un alto porcentaje ya presentaba MM. Esto refleja una pesquisa tardía, ya que el diagnóstico debiera realizarse cuando la discrasia de células plasmáticas aún se encuentra en fase de GMSR ${ }^{5}$.

La histopatología de RM fue exclusiva de MM, además, se presentaban con PN y PNN, sin síndrome nefrótico. Esta situación es esperable, ya que la proteinuria está determinada por un exceso de CL 


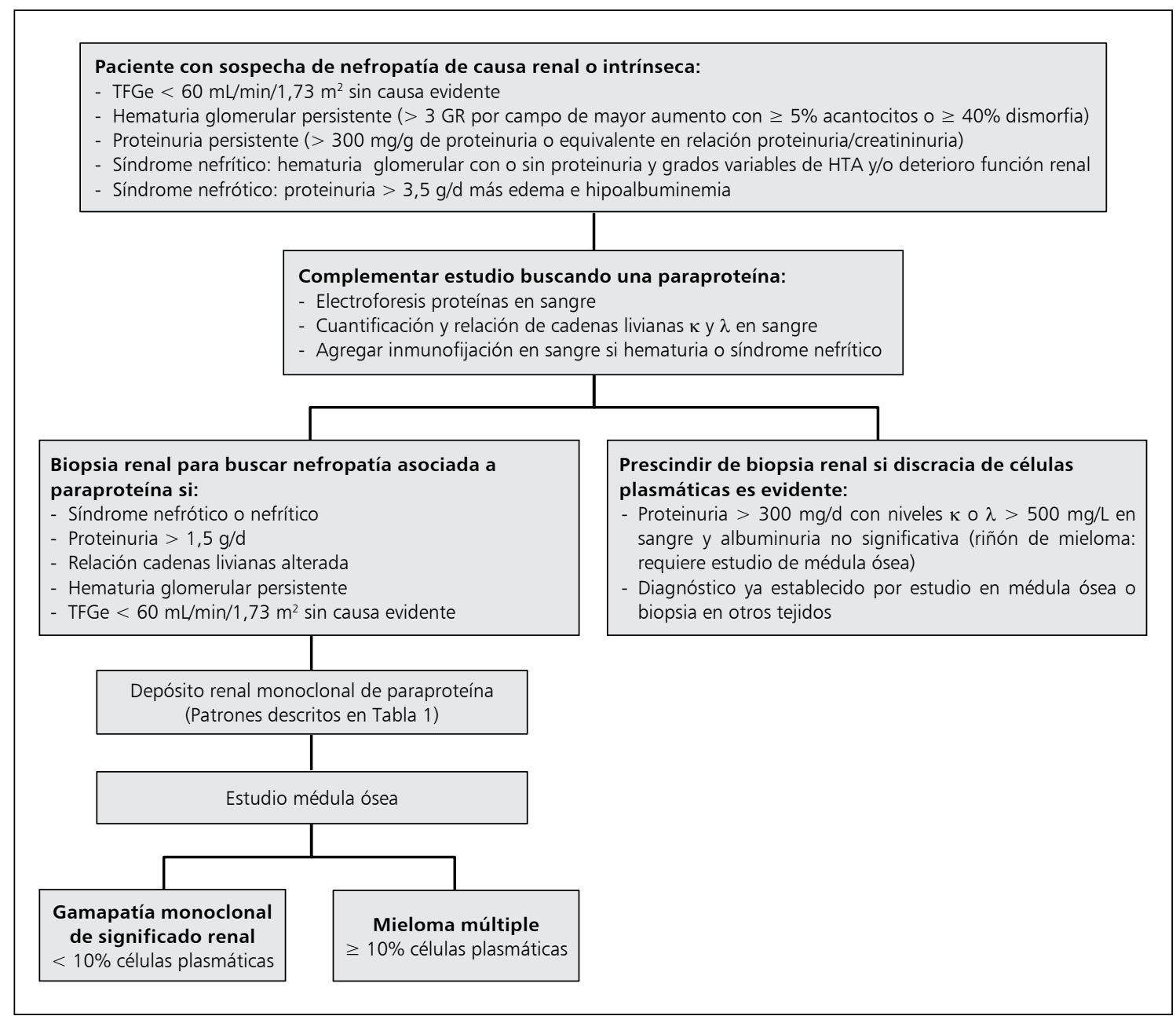

Figura 2. Algoritmo propuesto de estudio. GR: glóbulos rojos; HTA: hipertensión arterial; TFGe: tasa de filtración glomerular estimada.

que filtra en el glomérulo y no a una patología de este. Por lo tanto, en todo paciente con falla renal y proteinuria se debe buscar exhaustivamente una paraproteinemia.

En nuestra serie, la cuantificación de CL libres en sangre fue el examen más sensible para pesquisar pacientes con una paraproteinemia, concordante con los datos existentes en la literatura ${ }^{24}$, permitiendo descartar el diagnóstico en caso de resultar negativa. Le siguen en sensibilidad la inmunofijación de proteínas en sangre y luego en orina, por lo que pudiera preferirse la primera, dado su mayor facilidad de obtención. Menos sensible aun es la EFPS. Se describe que medir EFPS más CL libres en sangre permite identificar
$100 \%$ de los pacientes con MM, 96,5\% de aquellos con amiloidosis AL y $89 \%$ de los pacientes con $\mathrm{MGUS}^{24}$. Especialmente en estos últimos 2 grupos se encuentran los pacientes con GMSR. En pacientes con MIDD, 100\% presenta alteración en la relación de CL en sangre ${ }^{21}$, pero solo $50 \%$ de aquellos con PGNMID ${ }^{25}$. Los pacientes con PGNMID se presentan predominantemente con clínica de SNi y $96 \%$ tiene alteración en la IF en sangre ${ }^{25}$. La presencia de CL Lambda en sangre asociada a síndrome nefrótico fue un factor predictor del diagnóstico de amiloidosis renal, concordante con lo publicado en la literatura ${ }^{5,26}$.

En nuestra serie, independiente de la paraproteína en sangre, fue la CL la que tendió a producir 
la afección renal y no la CP. Entre los distintos tipos de GMSR, la presencia de CL Kappa se asoció con MIDD y PGNMID. En cambio, si había CL Lambda, fue más probable una amiloidosis renal. Por la importancia que tienen las CL en la GMSR, es trascendental que el estudio diagnóstico tenga un altísimo rendimiento para detectarlas.

Recomendamos que el estudio inicial de una paraproteinemia se realice con EFPS y CL en sangre; si en el cuadro clínico hay signos de nefritis, se deberá agregar una IF en sangre (Figura 2 ). Recientemente se evaluaron, en pacientes con MGUS, los hallazgos clínicos y de laboratorio que aumentaría la probabilidad de encontrar $\mathrm{GMSR}^{27}$ en estudio con biopsia renal, pesquisándose que tener una relación de cadenas livianas alteradas, hematuria y proteinuria mayor a 1,5 gramos en $24 \mathrm{~h}$ aumentaría su pesquisa, por lo que planteamos dichos criterios para considerar estudio de biopsia renal (Figura 2).

Dentro de las falencias de nuestro estudio están las propias de un estudio retrospectivo, con el inherente sesgo de información, un bajo número de pacientes y la imposibilidad de aplicar todos los tests diagnósticos al total de pacientes. Sin embargo, pese a las limitaciones, este estudio resalta la importancia de las nefropatías asociadas a paraproteínas, su mal pronóstico, la necesidad de tener una alta sospecha clínica al momento de estudiar un paciente y exámenes con adecuada sensibilidad para poder llegar a un diagnóstico. Solo así se podrá cambiar el curso natural de una enfermedad catastrófica, pero que, en la actualidad, tiene opciones de tratamiento y control.

\section{Referencias}

1. Wadhera R, Rajkumar V. Prevalence of Monoclonal Gammopathy of Undetermined Significance: A Systematic Review. Mayo Clin Proc. 2010; 85: 933-42.

2. Sipe JD, Cohen AS. Review: history of the amyloid fibril. J Struct Biol. 2000; 130: 88-98.

3. Kyle RA, Steensma DP. History of multiple myeloma. Recent Results Cancer Res. 2011; 183: 3-23.

4. Leung N, Bridoux F, Hutchison CA, Nasr SH, Cockwell $\mathrm{P}$, Fermand JP, et al. International Kidney and Monoclonal Gammopathy Research Group. Monoclonal gammopathy of renal significance: when MGUS is no longer undetermined or insignificant. Blood. 2012; 120: 4292-5.
5. Rosner MH, Edeani A, Yanagita M, Glezerman IG, Leung N. Paraprotein-Related Kidney Disease: Diagnosing and Treating Monoclonal Gammopathy of Renal Significance. Clin J Am Soc Nephrol. 2016; 11: 2280-87.

6. Perazella MA, Finkel KW. Paraprotein-Related Kidney Disease: Attack of the Killer M Proteins. Clin J Am Soc Nephrol. 2016; 11: 2256-59.

7. Bridoux F, Leung N, Hutchison CA, Touchard G, Sethi $\mathrm{S}$, Fermand JP, et al. Diagnosis of monoclonal gammopathy of renal significance. Kidney Int. 2015; 87: 698711.

8. Leung N, Bridoux F, Batuman V, Chaidos A, Cockwell $\mathrm{P}$, D'Agati VD, et al. The evaluation of monoclonal gammopathy of renal significance: a consensus report of the International Kidney and Monoclonal Gammopathy Research Group. Nat Rev Nephrol. 2019; 15: 45-59.

9. Shaik M, Al-Janadi A. Long term survival of monoclonal gammopathy of renal significance (MGRS): an analysis of Nhanes III. Blood. 2014; 124: 4849.

10. Steiner N, Göbel G, Suchecki P, Prokop W, Neuwirt $\mathrm{H}$, Gunsilius E. Monoclonal gammopathy of renal significance (MGRS) increases the risk for progression to multiple myeloma: an observational study of 2935 MGUS patients. Oncotarget. 2018; 9: 2344-56.

11. Leung N, Dispenzieri A, Fervenza FC, Lacy MQ, Villicana R, Cavalcante JL, et al. Renal response after high-dose melphalan and stem cell transplantation is a favorable marker in patients with primary systemic amyloidosis. Am J Kidney Dis. 2005; 46: 270-7.

12. Lorenz EC, Gertz MA, Fervenza FC, Dispenzieri A, Lacy MQ, Hayman SR, et al. Long-term outcome of autologous stem cell transplantation in light chain deposition disease. Nephrol Dial Transplant. 2008; 23: 2052-57.

13. Pinney JH, Lachmann HJ, Bansi L, Wechalekar AD, Gilbertson JA, Rowczenio D, et al. Outcome in renal Al amyloidosis after chemotherapy. J Clin Oncol. 2011; 29: 674-81.

14. Kourelis TV, Nasr SH, Dispenzieri A, Kumar SK, Gertz MA, Fervenza FC, et al. Outcomes of patients with renal monoclonal immunoglobulin deposition disease. Am J Hematol. 2016; 91: 1123-8.

15. Sigurdardottir EE, Turesson I, Lund SH, Lindqvist EK, Mailankody S, Korde N, et al. The Role of Diagnosis and Clinical Follow-up of Monoclonal Gammopathy of Undetermined Significance on Survival in Multiple Myeloma. JAMA Oncol. 2015; 1: 168-74.

16. Rajkumar SV, Dimopoulos MA, Palumbo A, Blade J, Merlini G, Mateos MV, et al. International Myeloma Working Group updated criteria for the diagnosis of multiple myeloma. Lancet Oncol. 2014; 15: 538-48.

17. Kumar S, Paiva B, Anderson KC, Durie B, Landgren O, 
Moreau P, et al. International Myeloma Working Group consensus criteria for response and minimal residual disease assessment in multiple myeloma. Lancet Oncol. 2016; 17: 328-46.

18. Kidney Disease Improving Global Outcomes (KDIGO) Clinical Practice Guideline for Glomerulonephritis. Kidney Int Suppl. 2012; 2: 1-143.

19. da Fonseca GS, Machado JR, Zago LBR, dos Reis MA, dos Reis M MLG, De Vito FB. Monoclonal gammopathy of renal significance: case report. Hematol Transfus Cell Ther. 2018; 40: 86-9.

20. Peña C, Schutz NP, Riva E, Valjalo R, Majlis A, López-Vidal H, et al. Epidemiological and clinical characteristics and outcome of monoclonal gammopathy of renal significance-related lesions in Latin America. Nephrology (Carlton). 2020; 25(6): 442-9. https://doi. org/10.1111/nep.13685.

21. Nasr SH, Valeri AM, Cornell LD, Fidler ME, Sethi S, D'Agati VD, et al. Renal monoclonal immunoglobulin deposition disease: a report of 64 patients from a single institution. Clin J Am Soc Nephrol. 2012; 7: 231-9.

22. Pozzi C, D’Amico M, Fogazzi GB, Curioni S, Ferrario F, Pasquali S, et al. Light chain deposition disease with re- nal involvement: clinical characteristics and prognostic factors. Am J Kidney Dis. 2003; 42: 1154-63.

23. Finkel KW, Cohen EP, Shirali A, Abudayyeh A. Paraprotein-Related Kidney Disease: Evaluation and Treatment of Myeloma Cast Nephropathy. Clin J Am Soc Nephrol. 2016; 11: 2273-9.

24. Katzmann JA, Kyle RA, Benson J, Larson DR, Snyder MR, Lust JA, et al. Screening panels for detection of monoclonal gammopathies. Clin Chem. 2009; 55: 1517-22.

25. Sethi S, Zand L, Leung N, Smith RJ, Jevremonic D, Herrmann SS, et al. Membranoproliferative glomerulonephritis secondary to monoclonal gammopathy. Clin J Am Soc Nephrol. 2010; 5: 770-82.

26. Motwani SS, Herlitz L, Monga D, Jhaveri KD, Lam AQ. Paraprotein-Related Kidney Disease: Glomerular Diseases Associated with Paraproteinemias. Clin J Am Soc Nephrol. 2016; 11: 2260-72.

27. Klomjit N, Leung N, Fervenza F, Sethi S, Zand L. Rate and Predictors of Finding Monoclonal Gammopathy of Renal Significance (MGRS) Lesions on Kidney Biopsy in Patients with Monoclonal Gammopathy [published online ahead of print, 2020 Aug 3]. J Am Soc Nephrol. 2020; ASN.2020010054. 